\title{
Complete Neurological Recovery after Severe Accidental Hypothermia: Two Cases and a Novel Algorithm Proposal
}

\author{
Khortnal Delvecchio ", Alexander L Marinica, Calvin Williams, Thomas Hanna, \\ Susan Seman, Zewditu Asfaw, Ingida Asfaw \\ Department of Surgery, Detroit Medical Center - Sinai Grace Hospital, Detroit, USA \\ *Corresponding author: khortnal@gmail.com
}

Received April 07, 2019, 2019; Revised June 02, 2019; Accepted June 04, 2019

\begin{abstract}
Primary accidental hypothermia results from endogenous thermoregulatory mechanisms being overwhelmed by environmental stressors. Vulnerable populations include the elderly, mentally ill, homeless, and chemically dependent. When core temperatures fall below $35^{\circ} \mathrm{C}$, the resulting metabolic derangements provoke cardiopulmonary compromise and dysrhythmia. Due to the human body's innate protective mechanisms there are reports of patients making complete neurologic recovery after exposure to extreme temperatures and prolonged cardiopulmonary arrest. We describe two cases from our institution demonstrating appropriate treatment methods for the two most severe stages of hypothermia. A 57-year-old male with a core temperature of $27^{\circ} \mathrm{C}$ who was successfully resuscitated with warm bladder and thoracic lavage and a 34-year-old female with a core temperature of $22^{\circ} \mathrm{C}$ that fully recovered after 5 hours of external cardiopulmonary bypass. From our experience and a review of the literature, we have developed a modified version of the Swiss staging model and incorporated it into a novel treatment algorithm for in-hospital adult accidental hypothermia patients presenting to urban community hospitals. The two example cases described highlight how appropriate utilization of this novel algorithm can lead to positive outcomes in otherwise critical clinical scenarios.
\end{abstract}

Keywords: hypothermia, cardiopulmonary bypass, extracorporeal membrane oxygenation, rewarming, case report, algorithms

Cite This Article: Khortnal Delvecchio, Alexander L Marinica, Calvin Williams, Thomas Hanna, Susan Seman, Zewditu Asfaw, and Ingida Asfaw, "Complete Neurological Recovery after Severe Accidental Hypothermia: Two Cases and a Novel Algorithm Proposal." American Journal of Medical Case Reports, vol. 7 , no. 6 (2019): 100-103. doi: 10.12691/ajmcr-7-6-2.

\section{Introduction}

Defined as unintentional core temperature below $35^{\circ} \mathrm{C}$, accidental hypothermia claims the lives of nearly 1500 individuals in the United States annually [1]. More recently, utilization of contemporary therapeutic techniques has allowed for successful resuscitation amid remarkably low core temperatures. Thermoregulation is normally mediated by the preoptic nucleus of the anterior hypothalamus, however primary accidental hypothermia occurs when environmental stressors overcome this regulation $[1,2,3]$. Using the Swiss staging model as a guide, treatment strategies can be devised for different core temperature thresholds based on their clinical implications [1]. We have modified the Swiss staging model to incorporate treatment levels appropriate for each hypothermia stage (Table 1). We then applied this modified classification toward a novel algorithm that allocates each hypothermia stage to the corresponding treatment level. We present two cases from a single urban community institution in which the appropriate treatment allocation resulted in positive outcomes. After reviewing these cases and the relevant literature, we propose a treatment algorithm for clinical assistance at similar institutions (Figure 1).

Table 1. Modified Swiss Staging Model for Hypothermia and Associated Treatments

\begin{tabular}{|l|c|c|c|c|}
\hline $\begin{array}{c}\text { Hypothermia } \\
\text { Stage }\end{array}$ & Classification & Temperature & $\begin{array}{c}\text { Clinical } \\
\text { Findings }\end{array}$ & Treatment \\
\hline HT I & Mild & $32-35^{\circ} \mathrm{C}$ & $\begin{array}{l}\bullet \text { Conscious } \\
\bullet \text { Shivering }\end{array}$ & $\begin{array}{c}\text { Level 1: } \\
\text { Passive }\end{array}$ \\
\hline HT II & Moderate & $28-31.9^{\circ} \mathrm{C}$ & $\begin{array}{l}\bullet \text { Impaired } \\
\text { consciousness } \\
\bullet \text { Not shivering }\end{array}$ & $\begin{array}{c}\text { Level 2: } \\
\text { Active } \\
\text { External }\end{array}$ \\
\hline HT III & Severe & $24-27.9^{\circ} \mathrm{C}$ & $\begin{array}{l}\bullet \text { Unconscious } \\
\bullet \text { Not shivering } \\
\bullet \text { Vitals present }\end{array}$ & $\begin{array}{c}\text { Level 3: } \\
\text { Active } \\
\text { Internal }\end{array}$ \\
\hline HT IV & Severe & $<24^{\circ} \mathrm{C}$ & $\begin{array}{l}\bullet \text { Unconscious } \\
\bullet \text { Not shivering } \\
\bullet \text { Absent vitals }\end{array}$ & $\begin{array}{c}\text { Level 4: } \\
\text { CPB or } \\
\text { ECMO }\end{array}$ \\
\hline $\begin{array}{l}\text { HT: Hypothermia, CPB: Cardiopulmonary bypass, ECMO: } \\
\text { Extracorporeal membrane oxygenation }\end{array}$ \\
\hline
\end{tabular}

Adapted and modified from "Accidental hypothermia," by D. J. Brown, H. Brugger, J. Boyd and P. Paal, 2012, New England Journal of Medicine, 367(20), p. 1930-8. 


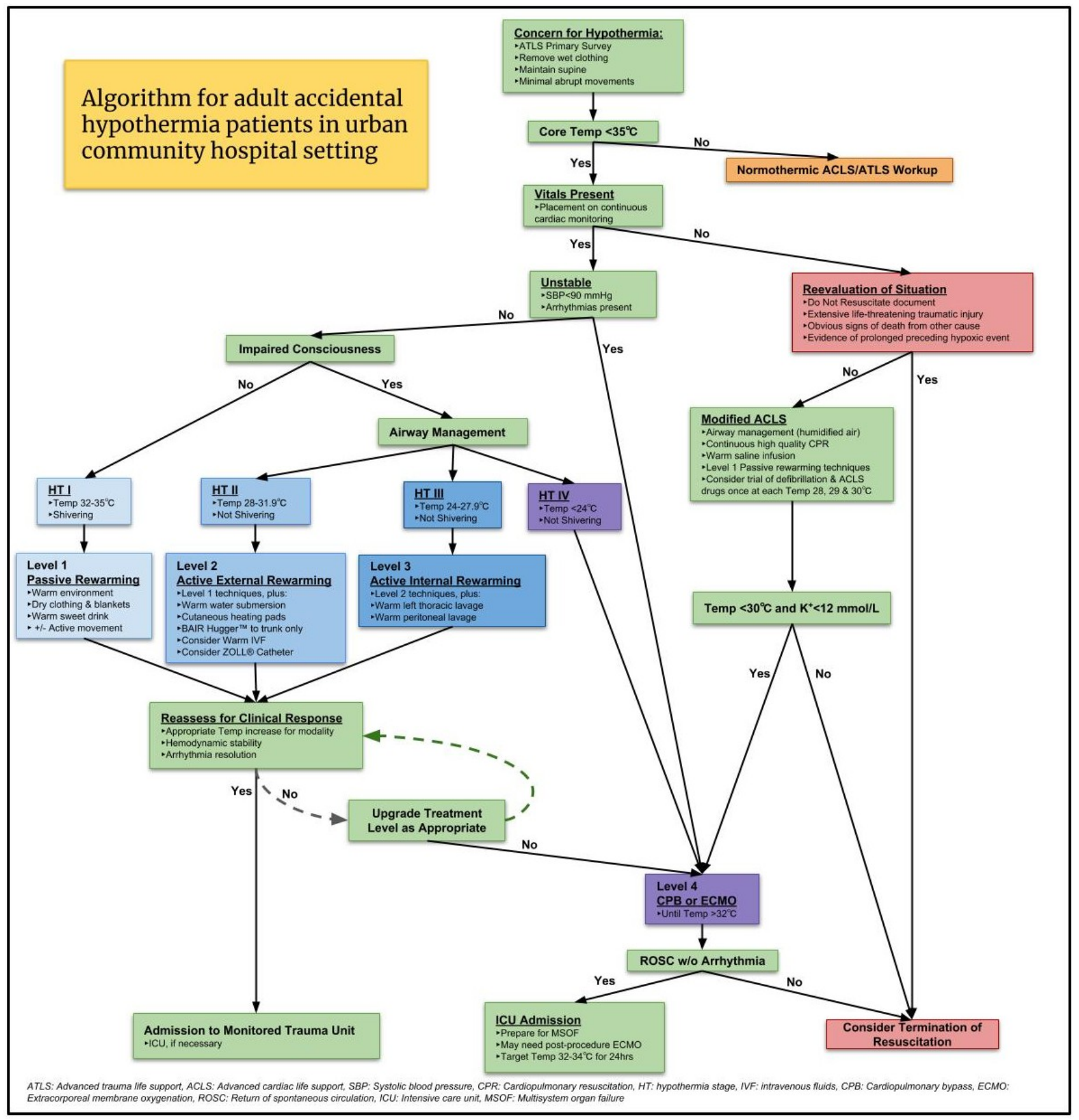

Figure 1. Proposed algorithm. Adapted and modified from “Accidental hypothermia," by D. J. Brown, H. Brugger, J. Boyd and P. Paal, 2012, New England Journal of Medicine, 367(20), p. 1930-8

\section{Case Reports}

Case 1 describes a 57-year-old male with a medical history significant for epilepsy who was found unresponsive in a puddle of water by emergency medical technicians (EMT) in February. Upon arrival to the hospital he was awake and responsive with a Glasgow Coma Scale (GCS) score of 12. In the resuscitation bay he had a measured systolic blood pressure in the 140's and was in atrial fibrillation. The patient's rectal temperature upon arrival was $27^{\circ} \mathrm{C}$, serum potassium level $3.7 \mathrm{mmol} / \mathrm{L}$, lactic acid $4.5 \mathrm{~mol} / \mathrm{L}$, and arterial $\mathrm{pH}$ 7.26. Due to his intact consciousness he was allocated to the Modified Swiss stage of HT I (Table 1) and thus Level 1 passive rewarming measures were initiated including warm blankets and application of a dry gown. Shortly after rewarming commenced, the patient experienced rapid mental deterioration to a GCS of 3 and he was immediately intubated. At this point with impaired consciousness and a core temperature less than $28^{\circ} \mathrm{C}$, the patient was upgraded to HT III and transported to the operating room (OR) for Level 3 active internal rewarming. In the OR, warm bladder irrigation and thoracic lavage via bilateral tube thoracostomies was initiated. After 3 hours, the patient reached a temperature of $32^{\circ} \mathrm{C}$, was hemodynamically stable without the need for cardiopulmonary resuscitation (CPR) or vasoactive substances, and he was transferred to the intensive care unit (ICU). He self-extubated the following day after a sedation vacation, was transferred out of the ICU on day 4 , 
and discharged home on day 11 with complete neurological recovery.

Case 2 involves a 34-year-old female with a medical history significant for HIV who was discovered unconscious outside in February by EMTs. The patient suffered cardiac arrest during transport, was subsequently intubated with a combitube, and CPR was initiated. In the resuscitation bay her combitube was exchanged for an endotracheal tube immediately and CPR was continued. The patient's rectal temperature upon arrival was $22^{\circ} \mathrm{C}$, serum potassium level $2.9 \mathrm{mmol} / \mathrm{L}$, lactic acid $5.0 \mathrm{~mol} / \mathrm{L}$, and arterial $\mathrm{pH}$ 6.98. From these clinical findings she was allocated to HT IV and was taken to the operating room for external cardiopulmonary bypass (CPB) via femoral cutdown, a Level 4 therapy. After temperature elevation to $34^{\circ} \mathrm{C}$ she required a single external defibrillation as well as initiation of vasoactive support. She subsequently reached $36.7^{\circ} \mathrm{C}$ and was weaned off $\mathrm{CPB}$ after a total of 5 hours. The patient was then transferred to the ICU where she was successfully extubated the following day. She was transferred out of the ICU on day 3 and discharged on day 5 with complete neurological recovery.

\section{Conclusion}

As described above, both cases presented here demonstrate identification and subsequent treatment allocation for severe hypothermic patients. It is vital to the patient's' prognosis that evaluation and diagnosis be completed immediately on arrival to the resuscitation bay to allow prompt allocation and treatment initiation. Our patient experiences above demonstrate the two most critical stages of accidental hypothermia, HT III and HT IV, requiring the most invasive treatments. To clarify exactly when each treatment method is warranted, we have created a Modified Swiss staging model complete with corresponding treatment levels (Table 1). From both our experience and a literature review, we propose a treatment algorithm for in-hospital adult accidental hypothermia patients presenting to urban community hospitals (Figure 1).

As is standard with all trauma admissions, treatment of a suspected hypothermic patient should begin with a primary survey. Additionally, supine positioning, wet clothing removal, and minimization of patient manipulation is often endorsed in the literature [3]. After the primary survey, core temperature should be measured via rectum or bladder. Though frequently lagging behind true core values, these methods are more accurate in cold settings than external type measurements [3,4]. For the unconscious/intubated patient, distal esophageal measurements closely correlate with pulmonary artery temperature $[1,4]$. If the patient is determined to be normothermic, the workup should follow normal trauma evaluation and treatment protocols. However, for core temperatures $<35^{\circ} \mathrm{C}$, these patients should be placed on monitors, and the algorithm continued to be followed.

The pathway continues by dissociating patients based on hemodynamic status, as this drastically alters the treatment course. Patients presenting without vitals or signs of life should be evaluated for other associated injuries and consideration should be given for termination of resuscitation measures. If efforts are to be continued,
CPR should be initiated as well as Level 1 rewarming therapy. Furthermore, a trial of Advanced cardiac life support medications and defibrillation can be instituted once temperature reaches $28^{\circ} \mathrm{C}[2,3,4]$. If temperature remains below $30^{\circ} \mathrm{C}$ and potassium $<12 \mathrm{mmol} / \mathrm{L}$, treatment should progress to $\mathrm{CPB}$ or $\mathrm{ECMO}$, otherwise the literature supports termination of resuscitative efforts [4]. Although no single objective prognostic marker exists, several retrospective studies have implicated serum potassium as most sensitive for mortality [3]. Values over $10 \mathrm{mmol} / \mathrm{L}$ indicate extensive cellular death, implying mortality prior to hypothermia $[3,5]$. Naturally, there is no documentation in the literature of which we are aware, of survival with a potassium $>12 \mathrm{mmol} / \mathrm{L}$, indicating its accepted use as a resuscitative cutoff and justifying its inclusion in our algorithm [4]. Of note, when core temperature reaches $32^{\circ} \mathrm{C}$ bypass techniques should halt, regardless of arrhythmia presence $[1,4]$.

Summarized in Table 1, stable patients without arrhythmias can be further classified by our Modified Swiss staging model with the appropriate treatment levels [1]. Hyperthermia stage I (HT I) is largely benign and can be treated with Level 1 passive rewarming methods that assist the body's endogenous mechanisms preventing further heat loss, including placement in a warm environment, dry clothing, humidified air inhalation, and ingestion of a warm sugary drink $[1,2,3,4]$. Any patient with signs of impaired conscious is automatically upgraded to at least HT II and airway management becomes paramount. HT II is indicative of the body's exhausted autonomic efforts and thus active external support is required, such as warm water immersion, cutaneous heating pads, heated blankets and Bair Huggers ${ }^{\mathrm{TM}}[1,2,3,4]$. At this stage warmed $\left(38-42^{\circ} \mathrm{C}\right)$ non-dextrose IV fluid may be carefully administered as well $[1,2,3]$. In HT III (described in case 1) the patient is in imminent danger of slipping into cardiac arrest and thus invasive active approaches are indicated: warmed IV fluids, and pleural or peritoneal lavage $[1,2,3,6]$. Despite mere modest results in recent studies, at HT II or III consideration may also be given to the placement of a centrally-located endovascular warming Zoll ${ }^{\circledR}$ catheter [6,7]. During treatment of HT I, II and III, it is important to continuously assess the patient's hemodynamic stability as well as their response to the therapy with appropriate core temperature elevation. These findings should then prompt reclassification of the patient and thus treatment level reallocation if necessary.

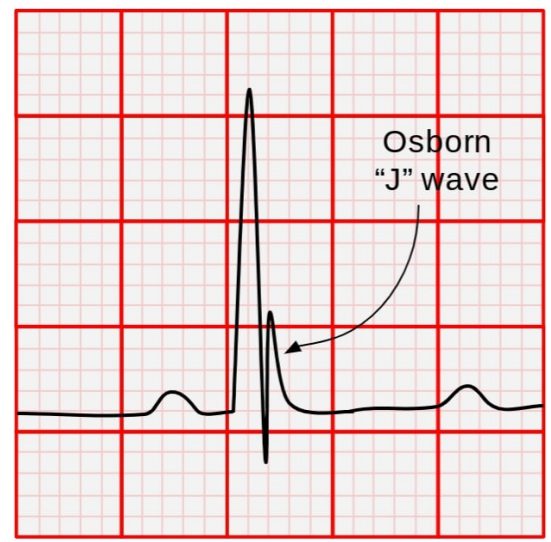

Figure 2. Depiction of Osborn "J" waves on electrocardiogram 
Finally, literature suggests that centers regard HT IV as clinically equivalent to hypothermia with cardiac arrest or a failure to progress from lesser stage therapies. Consequently, these patients require extracorporeal membrane oxygenation (ECMO) or CPB (as described in case 2) $[1,4,8]$. The debate comparing the merits of these two resuscitation methods is beyond the scope of this paper, however there is clear evidence that in the aforementioned clinical settings, these therapies should be utilized when available as they drastically improve survival $[1,3,4,8]$. Irrespective of major trauma or asphyxiation, death from hypothermia is due to cardiac dysfunction occurring as temperature rises $[1,2,4]$. During cooling, dehydration, acidosis, electrolyte disturbances from organ dysfunction leads to cardiac dysrhythmias [2]. Interestingly, despite the high prevalence, dysrhythmias frequently resolve without intervention amid rewarming [3]. A portion of patients, however, develop cardiac arrest requiring $\mathrm{CPR}$.

As temperature decreases, the brain (and other organs) utilizes less oxygen [4]. This incredible adaptation enables full neurologic recovery after extraordinarily long arrest states, reportedly up to 390 minutes [1,3]. As noted by Darocha et al. signs of imminent cardiopulmonary failure appear in a predictable stepwise fashion, with Osborn "J" waves (Figure 2) being the most classically described, and ventricular fibrillation being the most ominous (Table 2) $[7,9]$.

Table 2. Electrocardiographic Signs by Core Temperature

\begin{tabular}{|l|l|}
\hline Temperature & EKG Findings \\
\hline$<32{ }^{\circ} \mathrm{C}$ & Sinus bradycardia \& PR, QRS, QT prolongation \\
\hline$<30^{\circ} \mathrm{C}$ & Osborn "J" waves \\
\hline$<29^{\circ} \mathrm{C}$ & Atrial fibrillation \\
\hline$<28^{\circ} \mathrm{C}$ & Ventricular fibrillation \\
\hline $20^{\circ} \mathrm{C}$ & Asystole \\
\hline EKG: Electrocardiogram \\
\hline
\end{tabular}

At the conclusion of our algorithm we indicate that patients who have responded appropriately to therapy and are devoid of arrhythmias may be transferred to a monitored unit or ICU if necessary. Failure to respond despite maximal therapy takes our algorithm all the way to the right in which termination of efforts should be considered.

As is evident from our discussion, accidental hypothermia encompasses a vast array of clinical scenarios requiring coordination with a number of preand in-hospital teams. Its is our opinion that a clear understanding of the sequential order of evaluation, diagnosis and subsequent treatment is crucial to achieve favorable outcomes. Therefore, this pathway is one that the entire clinical team should be familiar with. We've applied accepted treatment conclusions from the literature toward a novel algorithm that we will implement in our institution, and hope to validate with future studies.

\section{Acknowledgements}

We thank all the clinical staff of the Detroit Medical Center - Sinai Grace Hospital, Department of Surgery for the support they rendered to us during the entire patient care and writing process.

\section{References}

[1] Brown DJ, Brugger H, Boyd J, Paal P. "Accidental hypothermia," N Engl J Med, 367 (20), 1930-8. Jan. 2012

[2] Petrone P, Asensio JA, Marini CP. "Management of accidental hypothermia and cold injury," Curr Probl Surg, 51 (10), 417-31. Jul. 2016.

[3] Kempainen RR, Brunette DD. "The evaluation and management of accidental hypothermia," Respir Care, 49 (2), 192-205. Feb. 2004.

[4] Paal P, Gordon L, Strapazzon G, Brodmann Maeder M, Putzer G, Walpoth B, et al. "Accidental hypothermia-an update: The content of this review is endorsed by the International Commission for Mountain Emergency Medicine (ICAR MEDCOM)," Scand $J$ Trauma Resusc Emerg Med, 24 (1), 111. Sep.2016.

[5] Mair P, Kornberger E, Furtwaengler W, Balogh D, Antretter H. "Prognostic markers in patients with severe accidental hypothermia and cardiocirculatory arrest," Resuscitation, 27 (1), 47-54. Jan. 1994.

[6] Klein LR, Huelster J, Adil U, Rischall M, Brunette DD, Kempainen RR, et al. "Endovascular rewarming in the emergency department for moderate to severe accidental hypothermia," $A m J$ Emerg Med, 35 (11), 1624-1629. May.2017.

[7] Turtiainen J, Halonen J, Syväoja S, Hakala T. "Rewarming a patient with accidental hypothermia and cardiac arrest using thoracic lavage," Ann Thorac Surg, 97 (6), 2165-6. Jun.2014

[8] Svendsen ØS, Grong K, Andersen KS, Husby P. "Outcome After Rewarming From Accidental Hypothermia by Use of Extracorporeal Circulation," Ann Thorac Surg, 103 (3) 920-925. Sep.2017.

[9] Darocha T, Sobczyk D, Kosiński S, Jarosz A, Gałązowski R, Nycz K, et al. "Electrocardiographic Changes Caused by Severe Accidental Hypothermia," J Cardiothorac Vasc Anesth, 29 (6), e83-6. Jul. 2015. 